\title{
EFFECTS OF GOP ON MULTIVIEW VIDEO CODING OVER ERROR PRONE CHANNELS
}

\author{
A.B Ibrahim ${ }^{1}$ and A.H Sadka ${ }^{2}$ \\ ${ }^{1}$ Department of Electronic \& Computer Engineering, \\ Brunel University, London, United Kingdom \\ Abdulkareem. Ibrahimebrunel. ac.uk \\ Abdul.Sadka@brunel.ac.uk
}

\begin{abstract}
In this paper, an investigation of the effects of group of pictures on H.264 multiview video coding content over an error prone environment with varying packet loss rates is presented. We analyse the bitrate performance for different GOP and error rates to see the effects on the quality of the reconstructed multiview video. However, by analysing the multiview video content it is possible to identify an optimum GOP size depending on the type of application used. A comparison is demonstrated for the performances between widely known H.264 data partitioning error resilience technique and multi-layer data partitioning technique with different error rates and GOP in terms of their perceived quality. Our simulation results turned out that Multi-layer data partitioning technique shows a better performance at higher error rates with different GOP. Further experiments in this work have shown the effects of GOP in terms of visual quality and bitrate for different multiview video sequences.
\end{abstract}

\section{KEYWORDS}

Multiview Video Coding, Group of Pictures, Error rates, Bitrate, and Video quality.

\section{INTRODUCTION}

Three-dimensional technology widely known as 3D technology has transformed many fields of discipline such as entertainment, communications, medicine and many more. 3D can be perceived in a number of different ways. We shall restrict our understanding to just multiview video coding in this paper. Generally, the main concept of video coding is to exploit the statistical correlation between consecutive frames. The MVC extension of the H.264/AVC exploits these similarities between frames, simplifies the decoding process, and advances new features that are specific to multiview video coding [1]. Multiview video coding has emerged as advancement in video coding technology. The multiview video coding system enables efficient encoding of sequences that are captured from different cameras at different locations at the same time. The H.264 MVC codec takes as an input several synchronized bitstream that are captured from several different cameras and generate a single bitstream as an output for storage or transmission [2]. The work in [3] gives a detailed overview of the MVC standard. The structure of MVC is defined by a concept known as matrix of pictures (MOP). In this technique, each row consists of a group of pictures (GOP) normally captured by the base view and each column represents the time domain of the video.

David C. Wyld et al. (Eds) : SAI, CDKP, ICAITA, NeCoM, SEAS, CMCA, ASUC, Signal - 2014 pp. 135-150, 2014. (C) CS \& IT-CSCP 2014

DOI : $10.5121 /$ csit.2014.41114 


\section{BACKGROUND}

The H.264/AVC international standard [4] has specifies a coding standard of video data. H264 defines three picture types namely I-frame, P-frame, and B-frame. In a standard reference multiview video encoder all the pictures in a multiview video are encoded with a fixed GOP length depending on the settings and applications. The arrangement of these three picture types in a sequence is distributed statistically within the group-of pictures. The special type of I-frame at the beginning of a sequence also known as an IDR frame serves as an entry point to facilitate random seeking or switching between channels. This can further be used in providing coding robustness to transmission errors [5] which are only coded with moderate compression to reduce the spatial redundancies in the multiview video sequence. I frames are generally larger than $\mathrm{P}$ and $B$ frames which means the less you have the longer the GOP size and the more compression you can get. But in multiview video content transmission especially in error prone channels, very long GOP can have an adverse effect of propagating error spatially, temporally and in interview direction. $\mathrm{P}$ frames are coded in an efficient way through the concept of motion compensation from either a past I or P frame which are mostly used as a reference to predict further. B frames have a very high compression ratio which requires the presence of both a past and future reference pictures for motion compensation.

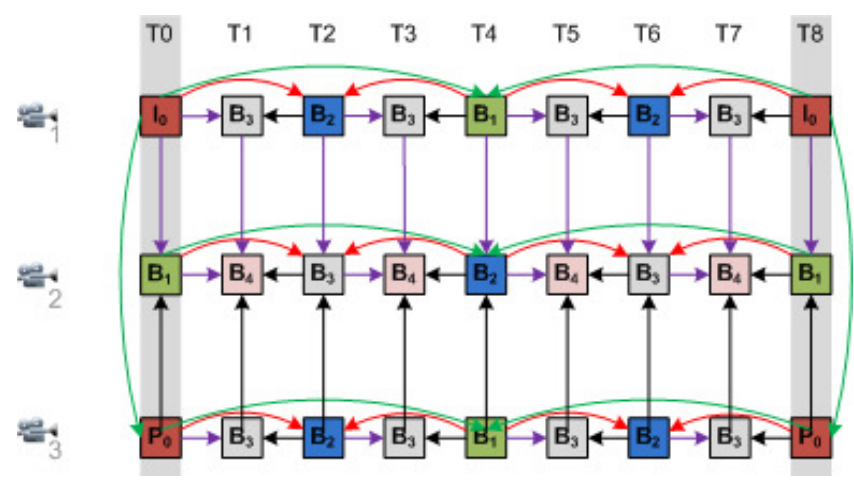

Figure 1. MVC prediction structure with GOP size of 8 [6].

Fig. 1 depicts a multiview video coding prediction structure with GOP size of 8 where I, P, and B represents the encoding of pictures in intra mode, predicted mode and bi-predicted mode respectively. The compressed multiview video data is highly sensitive to noise and information is loss due to the removal of statistical and subjective redundancy in the video by the compression scheme [7]. H.264/AVC employs variable length coding (VLC) in order to achieve higher compression gain. This type of predictive coding technique makes the video data highly sensitive to bit errors, and the effects of errors on the perceptual video quality can be quite severe. Thus, it is necessary to provide an effect technique and configuration settings that can make the MVV bitstream more robust to transmission error and to improve the visual quality of the reconstructed multiview video [8]. The effectiveness of H.264/AVC coding depends on many coding parameters one of which is GOP size and its internal organization [9]. Most standard reference H.264 codecs use a fixed size for the GOP to encode video sequences. The GOP size can have different values as specified by the standard, however, once a given size is chosen, it becomes applicable to the entire coding process and the corresponding standard decoder can be able to sort out the positioning of these frames during decoding process. 


\subsection{Concept of Data Partitioning in H.264/AVC}

The H.264/MPEG-4 AVC standard is established to represent complete video information in a much lower level called the slice. A H.264 video slice consists of an arbitrary integer number of successive macroblocks that represent different types of video data [10]. Slice header conveys information that is common to all the MBs in the slice such as the slice types which determine which MBs types are allowed, and frame number that the slice corresponds to, reference picture settings and default quantization parameter. The slice data section consists of a series of MBs that make a slice.

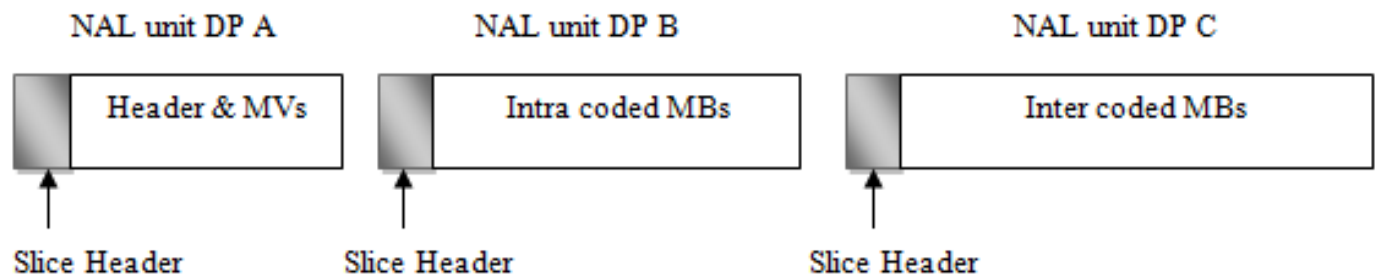

Figure 2. H.264/AVC Slice layout with data partitioning

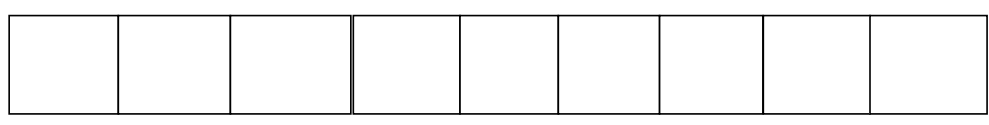

Figure 3. Multi-Layer data partitioning technique

In DP technique, MV and the residual information are separated by a boundary marker which is a uniquely decodable codeword. The codeword indicates the end of header information in a slice and the beginning of residual information [11]. Recent study on the concept of DP can be found in [12]. Data partition, nonetheless, creates more than one bit string (partitions) in every slice, and rearrange all symbols of a slice into a separate partition that have a close semantic relationship with each other Fig. 3. In H.264/AVC, when data partition is enabled, each slice of the coded bitstream is divided into three separate partitions with each of the partitions being from either type A, type B or type C partitions. Type A partition consists of header information, Quantization parameter $(\mathrm{QP})$, Macroblock type, reference indices and motion vectors. The intra partition also called type B consists of the Discrete Cosine Transform (DCT) intra coded coefficients and the inter partitions also known as type $\mathrm{C}$ partitions contain DCT coefficients of motion compensated Inter-frame coded MBs. Type $\mathrm{C}$ partition in many cases is the biggest partition of a coded slice and yet the least sensitive to error because its information does not synchronise the encoder and the decoder [13]. Each partition is placed in a separate Network Abstraction Layer (NAL) unit and may be transmitted separately over a network. The use of both Types B and C will require a type A partition and not vice-versa.

\subsection{Previous Work}

The implementation of data partitioning technique for MVC is presented in [14]. A video slice without any ER mechanism may be affected by transmission errors that can lead to the loss of the entire information within the slice. Implementation of error resilience techniques such as data partitioning in the JMVC reference software is necessary because there is no provision for any ER technique in the MVC in the reference software. Therefore, in order to analyse the performance of MVC in error-prone networks, implementation of a valid error resilience technique such as data partitioning as shown in Fig. 2 is employed and implemented in the JMVC 8.5 reference software. 
From the H.264 data partitioning technique, a video slice can be recovered when either partitions $\mathrm{B}$ or $\mathrm{C}$, or both, are affected by transmission errors as long as the partition $\mathrm{A}$ is not affected or lost as a result of losing the header and motion information contained therein. It has been observed that the performance of H.264/AVC data partitioning technique in MVC is not too encouraging and further error performance improvements can be made through the introduction of the proposed multi-layer data partitioning technique depicted in Fig.3.

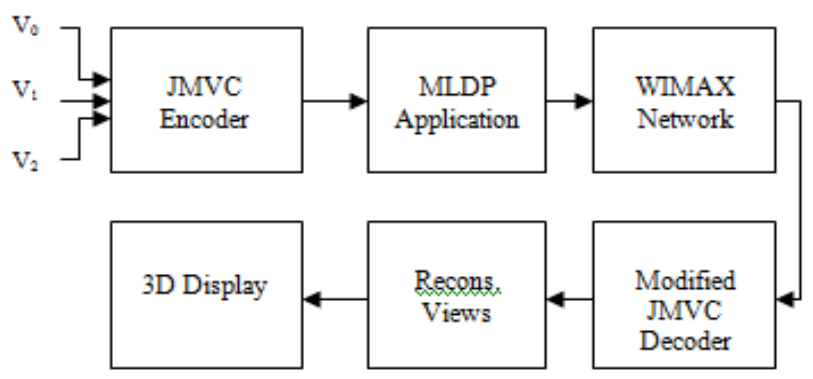

Figure 4. Flow diagram of the Multi-Layer DP technique

\subsection{Multi-Layer Data Partitioning Technique}

In an attempt to make the MVV bitstream more error resilient to the transmission errors in an error prone network, we propose a technique that can create two-layer of partitioning for each slice in the multiview video bitstream. The general concept of the technique is illustrated in Fig.4.The assembled multiview video bitstream is parsed in the developed Multi-Layer DP application for increased robustness against the transmission errors before transmitting over the wireless network. The partitioned bitstream is received by the modified JMVC reference decoder in order to decode and reconstruct the multiview video bitstream for viewing at the display. Multi-Layer DP adopts a mechanism that restructures a video slice as shown in Fig. 3. $\mathrm{A}_{0}$ partition consists of the header information of frame 0 from view 0 , and $A_{1}$ partition consists of the header and motion information of frame 1 from view 1 and $\mathrm{A}_{2}$ partition consists of the header and motion information of frame 2 from view 2. $\mathrm{B}_{0}$ consists of the residual information of intra coded MBs of frame $0, B_{1}$ consists of the residual of intra coded MBs in frame 1 and $B_{2}$ consists of the residual of intra coded $\mathrm{MBs}$ of frame 2 and $\mathrm{C}_{0}$ is an empty partition, $\mathrm{C}_{1}$ consists of residuals of inter coded $\mathrm{MBs}$ and $\mathrm{C}_{2}$ consists of the residual of inter coded MBs of frame 2 and in that sequence it continues till nth view and nth last slice of the multiview bitstream.

Note that, partition $\mathrm{C}_{0}$ is empty because there is no residual information of inter-coded MB's in frame 0 which is an intra-coded frame. I-frames are self-referential and do not require any sort of information from other frames to be predicted, so it consists of only intra coded MBs. The H.264 compliant encoder needs not to send empty partitions to the decoder because a standard H.264 decoder will assume missing partitions are empty partitions and are designed to handle the multiview bitstream accordingly [15]. During the decoding process of the MLDP bitstream, the decoder is modified to cope with the lost video data due to errors in the wireless channel.

The effects of displaying a frame reconstructed from a corrupted data can adversely degrade visual perception by introducing artefacts. In order to support the MLDP technique more effectively and to minimise the effects of channel errors in the multiview video bitstream, a simple and commonly known error concealment technique is employed and developed in the JMVC 8.5 reference decoder. Lost data in the bitstream can be concealed by copying the information from previously received error free slices. Frames that are generated by copying related video data in order to replace lost information are not always perceptually noticeable by a viewer which is an advantage of this technique especially in low-activity scenes [16]. In our 
approach, we are able to support multi-layer data partitioning technique with improved quality by employing frame copy error concealment which works fairly well with MVC and is simple to implement; however, there are more complex techniques that use an elaborate approach to exploit the redundancy within the video frame in order to come up with a more efficient estimate of the lost data [3].

Time first coding Fig. 5 is a MVV bitstream format representation that allows all views to be encoded and then assembled in a time domain for suitable transmission. The decoder on the other side can receive and reorder the bitstream in the right decoding order, which can allow it to decode all the pictures in different views in the same time domain and display the videos in the correct order.

Time first coding supports the implementation of frame copy error concealment in MVC. That is because of the display nature of all the frames across the views in the same time domain, which makes it easier to conceal missing pictures from previously received pictures in the reference list.
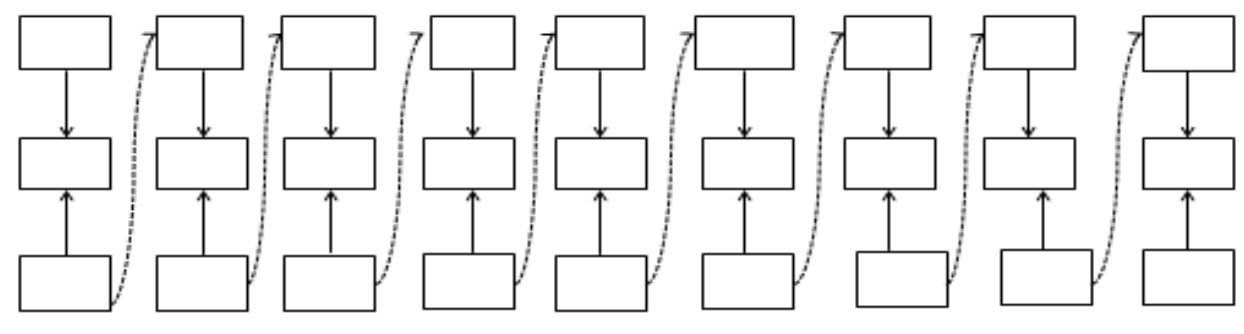

Figure 5. Time first coding [1].

Currently, the MVC reference decoder only accepts H.264 compliant bitstream and does not support the decoding of erroneous coded video sequence. In order to be able to decode the corrupted multiview video bitstream, the H.264/AVC frame copy error concealment technique is implemented in the JMVC reference decoder to adapt and cope with the losses within the bitstream. Frame copy error concealment technique is simple and usually quite effective in a video content where the motion is not large [17]. In Addition, the JMVC 8.5 reference codec has two types of reference frame lists that is also part of the standard and can be used to support frame copy error concealment in MVC. The first list is a reference list 0 which can be used for both $\mathrm{P}$ and $\mathrm{B}$ frames while reference list 1 is only applicable for B-frames. The main difference between the two reference lists is that list 0 utilizes the temporally earlier key frames (I or P) within the GOP in a sequence while in the case of the reference picture list 1; it utilizes temporally closer reference frames which can be a B frame [18]. Conceptually, reference list 1 can ensure smoother pictures because the frame to be copied is nearer to the picture to be reconstructed.

\subsection{Proposed decoding scheme}

H.264/AVC Frame copy error concealment technique is implemented in the JMVC reference decoder and further modified to decode the Multi-layer DP bitstream with losses as earlier discussed in the previous section. The technique is optimized to reconstruct all the views successfully from the multiview coded bitstream with a higher level of quality in conformance with the standard [19].

Part of the reasons and motivation to adopt frame copy error concealment technique in our work is its convenience to replace missing pictures especially in the case of packet loss network. 
The flowchart in Fig. 6 illustrates the implementation of frame copy error concealment technique. The technique can conceal lost information in the MVV bitstream with an improved perceptual quality based on some experimental results presented later in the paper.

When the ML data partitioned bitstream is transmitted over the network and is received, it is first buffered and rescheduled back to the standard H.264 DP format for processing. Note that, the multi-layer data partitioning technique employed during source coding is only to make the multiview video bitstream more resilient to channel errors during transmission or streaming over the simulated wireless network. After successfully delivering the bitstream across the network, then the received bitstream is rescheduled back to the standard H.264 data partitioned format for decoding.

The decoder checks if the buffer is full then all the frames are sent directly for decoding. Also, note that all the slices are partitioned into three different partitions encapsulated into VCL NAL units of DP A, DP B and DP C respectively. The decoding of these types of slices is such that the loss of one partition might make another partition useless. In order to correctly decode partitions B and C, it is important for the H.264 standard compliant decoder to know how each and every macroblock is predicted within a slice. This information is stored in partition A as part of header information. Therefore, loss of partition A can render partitions B and C useless even when correctly received and decoded. Partition A does not necessarily require the information from partition B and C to be correctly decoded.

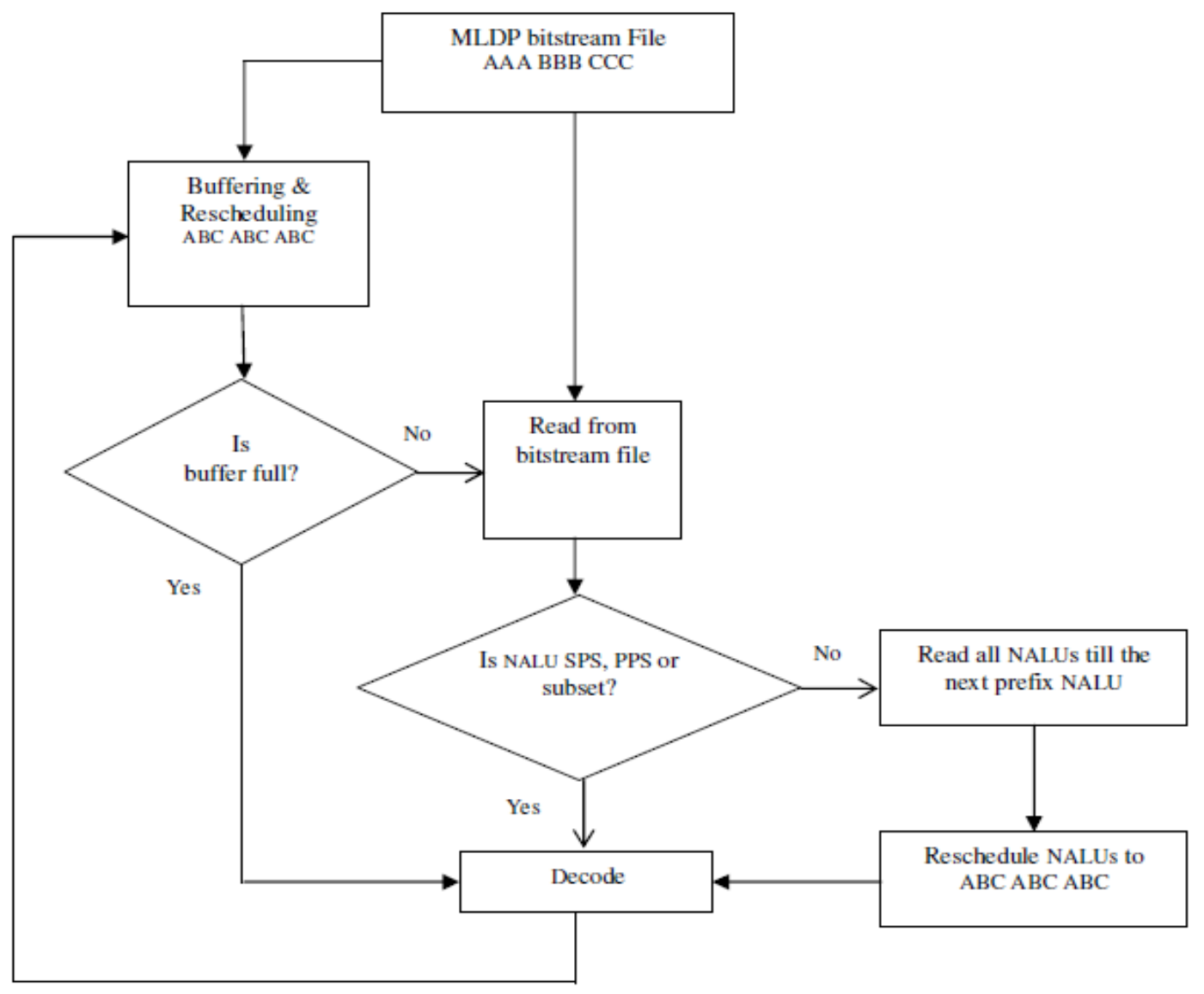

Figure 6. Decoding scheme for erroneous MVV bitstream 
So, if only partition A is correctly received then the error concealment algorithm can utilize useful information such as motion vectors to reconstruct the slice. However, if partition A is lost regardless of whether partition $\mathrm{B}$ or/and $\mathrm{C}$ is/are received. The frame copy error concealment is invoked by the decoder to replace the missing picture information by a previously received picture in the reference list. If the buffer is empty, then the NAL units are read from the MVV bitstream and the decoder determines whether it is a non-VCL NAL unit or VCL NAL unit? All non-VCL NAL units are sent directly for decoding while the VLC NAL units are all read until the next prefix NAL unit is detected and are rescheduled to the H.264 format before decoding. The whole process is restarted again through a looping system.

\section{SIMULATION}

To show the performance of 3D MVV bitstream over a wireless error-prone network, a number of coding and transmission experiments and simulations are performed in both JMVC 8.5 reference software and OPNET 16.1 network simulator [20]. This section describes the conditions used in the experimental setup.

\subsection{Video Encoder Settings}

Different MVV test sequences were used in the experiment and simulations such as Ballroom, Exit, and Vassar. Frame size of $640 X 480$, Frame rate $=25 \mathrm{f} / \mathrm{s}$, Number of Frames per view $=250$, and Quantization parameter (QP) was carefully selected and set to 31 and an intra-coded frame was inserted every 13th frame in order to limit the temporal error propagation. The JMVC 8.5 reference software and simulations were configured as in [18]. Symbol mode is set on Content Adaptive Variable Length Coding (CAVLC) to support the DP in the extended profile, also one slice per NAL unit is considered as part of the H.264/AVC network friendly design [21]. Three views of each of the MVV test sequences were considered to generate the MVV bitstream used for transmission over the simulated network.

\subsection{Transmission Simulation Setup}

This section describes some of the necessary conditions and parameters used in the network simulation setup. The robustness of MLDP against channel errors is demonstrated by examining the performance of the MVV bitstream transmitted over a WIMAX simulated channel under varying channel conditions and error rates. Multiple Subscriber Stations (SS) that represents source and destination nodes are configured to share a common Base Station that is connected to the core network through an IP backbone. The WIMAX model in OPNET does not have a direct approach to upload and introduce errors in an MVV bitstream file. Trace file of the MVV bitstream need to be generated first and simulated across the network. Transmission error distribution formats have been developed for different error rates in the model. The network simulation methodology is similar to the work in [22]. The MVV bitstream is decoded and evaluated after being received by the application client for different error rates in the network. The objective quality of the reconstructed videos is measured and analysed in terms of Peak Signal to Noise Ratio (PSNR) which is a widely known objective metric used to measure the reconstructed video quality [23].

\subsection{Experimental Results and Analysis}

This section describes the performance evaluation and results of the effects of GOP size on multiview video bitstream over the wireless network. The values of GOP sizes used in the experiments are 4, 8, 12, and 16 respectively. Also, the error rates used are $0 \%, 1 \%, 5 \%, 10 \%$, 
$15 \%$, and $20 \%$ respectively. For every GOP size and error rate considered, ten different simulations are conducted, and the average results are generated. The perceptual quality of each reconstructed view is measured in terms of peak signal to noise ratio (PSNR) for all the different simulations and error rates used in the experiment. Ballroom sequence experimental values for perceptual quality are generated in table 1 for different loss rates and GOP sizes

In Fig. 6, we evaluate both the H.264 DP and the multi-layer DP method for different error rates and GOP size. We have observed for different runs in our simulations that high error rate $(20 \%)$ multi-layer DP has a better and improved quality performance than the H.264 DP technique in many instances. Note that, video coding works either as fixed quality and variable bitrate and vice-versa.

In this experiment for various quality levels with GOP of 4, 8, 12 and 16, corresponding constant bitrates of $1909.69 \mathrm{~kb} / \mathrm{s}, 1619.76 \mathrm{~kb} / \mathrm{s}, 1527.94 \mathrm{~kb} / \mathrm{s}$, and $1374.75 \mathrm{~kb} / \mathrm{s}$ are respectively reported for ballroom test sequence Fig. 7.

In Fig. 8 and 9, the results of the experiment have revealed that a small number of GOP size means more I frames. This can have a tendency to consume more of bits because of the frequent occurrence of intra frames within the GOP. However, having more I-frames increases the multiview bitstream size. It can have a tendency of reducing the efficiency of the multiview video coding. Different applications can have different GOP requirements such as real time and offline applications each having a different latency or delay requirement [24].

In Fig. 10, the results obtained illustrate that lower GOP size can give a better perceptual quality in the multiview video. This is because low GOP means more intra frames within the GOP with less prediction error which can result in a higher video quality. In video communications overerror prone environment, trade-off between perceptual quality and bitrate consumption is important and necessary [25]. In most cases, applications requiring a high level of quality in an error-prone network can have a higher bitrate in order to make the MVV bitstream more resilient to channel noise and that result in visual quality improvement. [26]

\subsubsection{Objective and Subjective analysis}

Table 1. Numerical simulation results

\begin{tabular}{|l|l|l|l|l|}
\hline \multicolumn{2}{|l|}{ Ballroom GOP4 } & \multicolumn{2}{l|}{ Ballroom GOP8 } \\
\hline PLR $(\%)$ & H264 DP $(\mathrm{dB})$ & H264 ML $(\mathrm{dB})$ & H264 DP $(\mathrm{dB})$ & H264 ML(dB) \\
\hline 0 & 35.45 & 35.45 & 35.16 & 35.16 \\
\hline 1 & 34.53 & 34.93 & 34.67 & 34.72 \\
\hline 5 & 28.54 & 28.90 & 30.28 & 27.97 \\
\hline 10 & 24.73 & 24.37 & 26.82 & 24.96 \\
\hline 15 & 21.04 & 22.93 & 21.35 & 21.90 \\
\hline 20 & 18.65 & 20.04 & 18.09 & 19.04 \\
\hline
\end{tabular}

\begin{tabular}{|l|l|l|l|l|}
\hline \multicolumn{4}{|l|}{ Ballroom GOP 12} & Ballroom GOP 16 \\
\hline PLR $(\%)$ & H264 DP $((\mathrm{dB})$ & H264 ML $(\mathrm{dB})$ & H264 DP $((\mathrm{dB})$ & H264 ML $(\mathrm{dB})$ \\
\hline 0 & 34.99 & 34.99 & 34.83 & 34.83 \\
\hline 1 & 34.74 & 32.83 & 34.38 & 33.41 \\
\hline 5 & 30.42 & 30.10 & 30.42 & 31.82 \\
\hline 10 & 24.24 & 24.22 & 24.61 & 25.59 \\
\hline
\end{tabular}




\begin{tabular}{|l|l|l|l|l|}
\hline 15 & 20.94 & 21.63 & 19.23 & 22.52 \\
\hline 20 & 18.23 & 20.09 & 16.01 & 19.17 \\
\hline
\end{tabular}

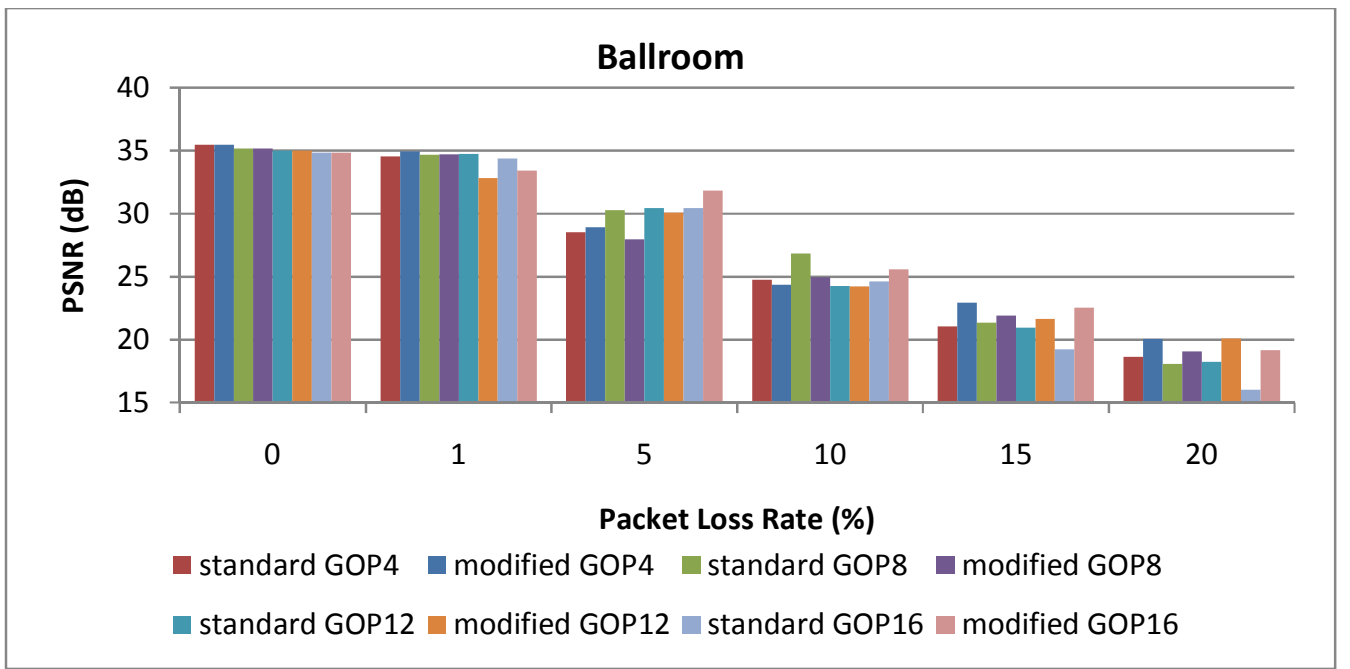

Figure 6. Quality evaluation for different error rates and GOP sizes

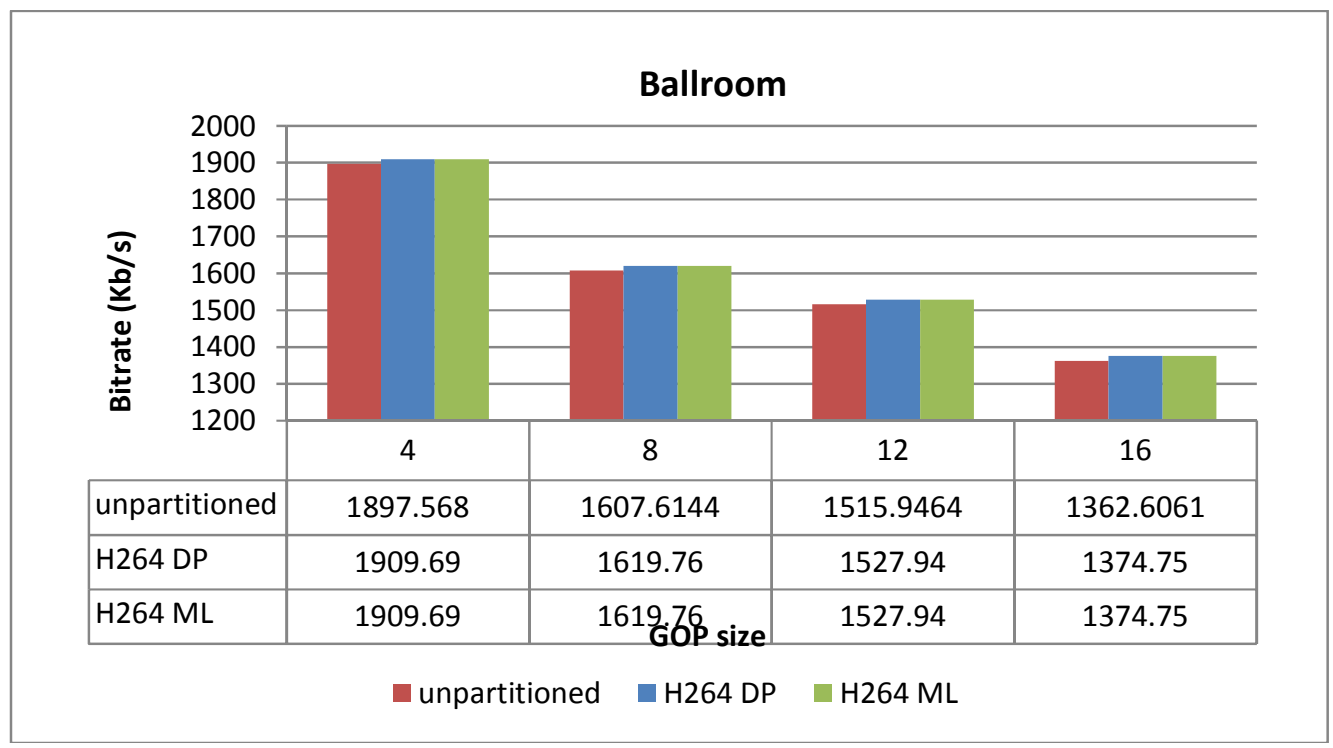

Figure 7. Bitrate performance for different GOP sizes 


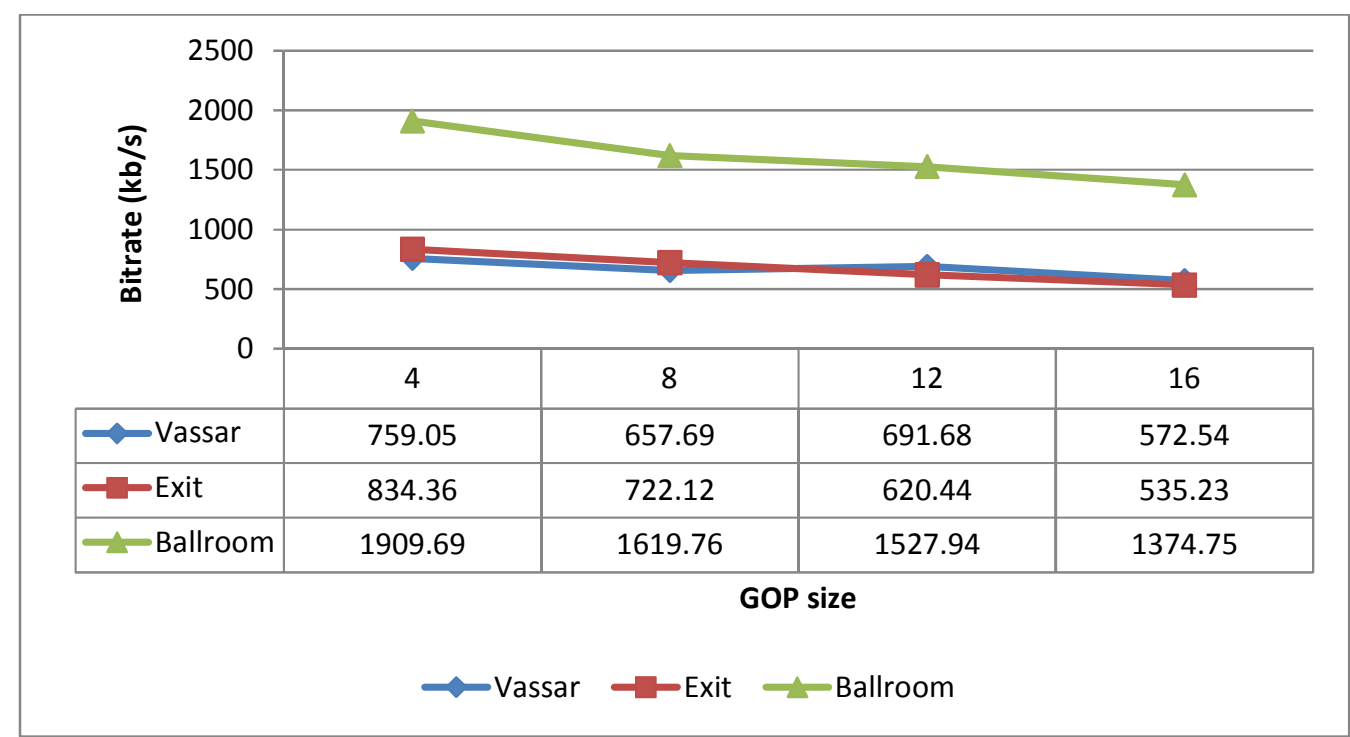

Figure 8. Bitrate performance for different GOP and test sequences

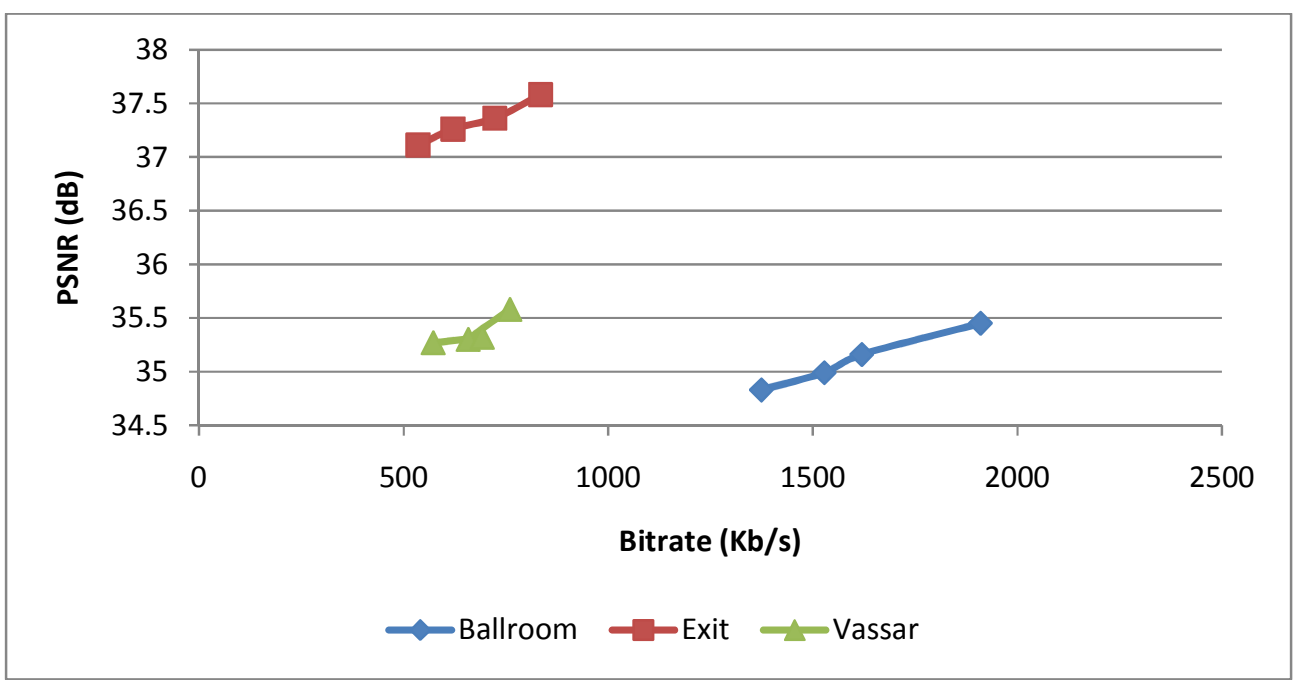

Figure 9. Quality and bitrate evaluation for different test sequences 


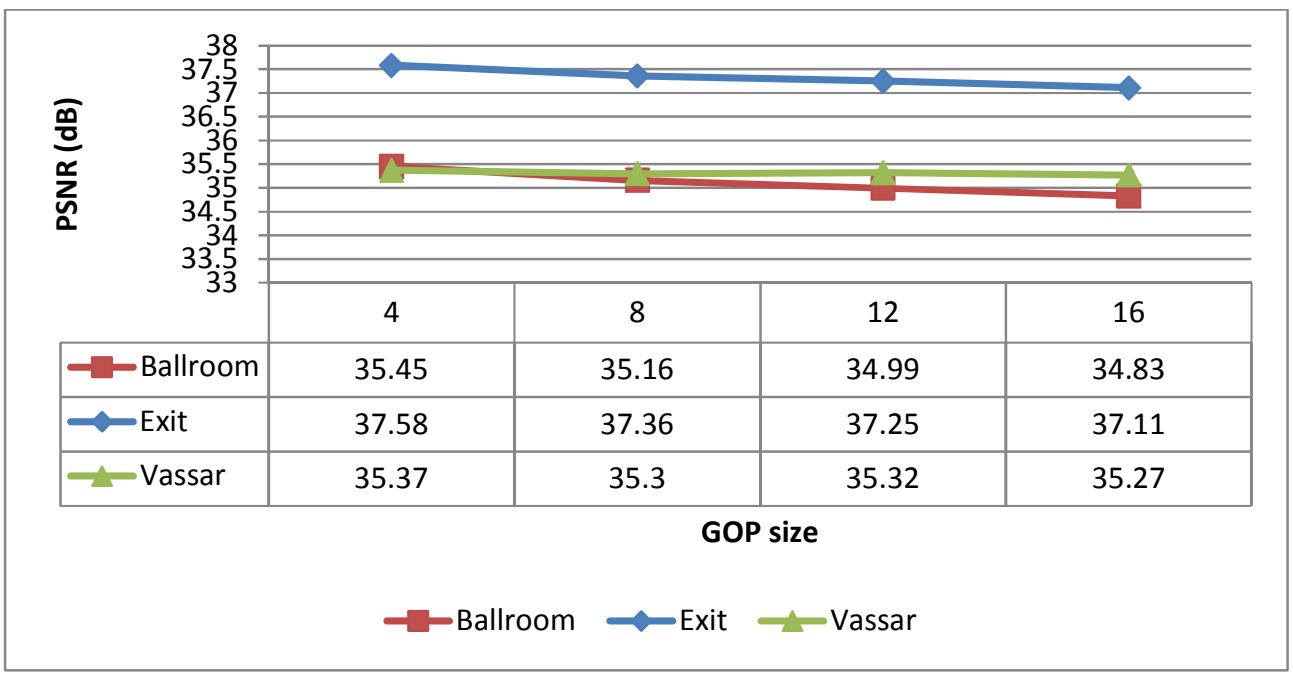

Figure 10. Quality evaluation for different GOP sizes and test sequences

The subjective results are presented for ballroom sequence for different views in Figures 11-13. In the demonstration, frame 121 is chosen from each view at $20 \%$ loss rate and a GOP of 16 . It can be observed that Multi-Layer DP technique can improve the perceptual quality performance than H.264 DP technique. The greyscale effect in Multi-layer DP technique is completely removed. We can observe closely in the Multi-layer DP that these frames are not reconstructed with the best quality when compared with the original frames. This is because of the high error rate used in the network simulations and the inability of the frame copy error concealment to recover high losses. At such error rate of $20 \%$ and GOP of 16, the multi-layer DP technique could recover most of the lost video information with improved quality compared to H264 DP technique at the same error rate and GOP size. It is important to analyse the effects of error propagation within a GOP of the multi-layer data partitioned bitstream. In hierarchical GOP like the one in multiview video coding, the reference decoder uses the I-frame in the base view and the anchor frames in the non-base view either directly or indirectly as reference frames for all other frames with the GOP. If an error occurs in the I-frame of view 1, it can result to artefacts that can continue to propagate throughout the GOP structure. The effect can be experienced in both temporal and interview manner until the next random access point. At this point, the decoder refreshes with the next intra coded frame in view 1 or the anchor frames in either view 2 and 3. It has been noticed that losses within the I-frame that does not affect the header information such as intra coded MBs coefficient can also propagate errors throughout the GOP. P-frames are coded using motion compensation prediction from previous reference frames. From Fig. 1, anchor frame such as the one in view 3 is forward predicted from the I-frame in view 1, subsequent prediction of other non-anchor frames in both view 3 and view 2 takes reference from their preceding P-frame. Any form of loss in this frame can further propagate error through the remainder of the GOP until the next refresh frame is received within the multi-layer partitioned bitstream. It can be highlighted that the impact of P-frame or anchor frame of view 3 can be almost as significant as losing an I-frame due many of interdependencies from other frames. Due to the hierarchical nature of MVC bitstream, anchor frame in view 2 that is interview predicted from view 1 and view 3 is used to predict other non-anchor frames temporally within the GOP. So the effect of error is limited to view 2 only and less severe than I and P-frames in the multiview video bitstream. 

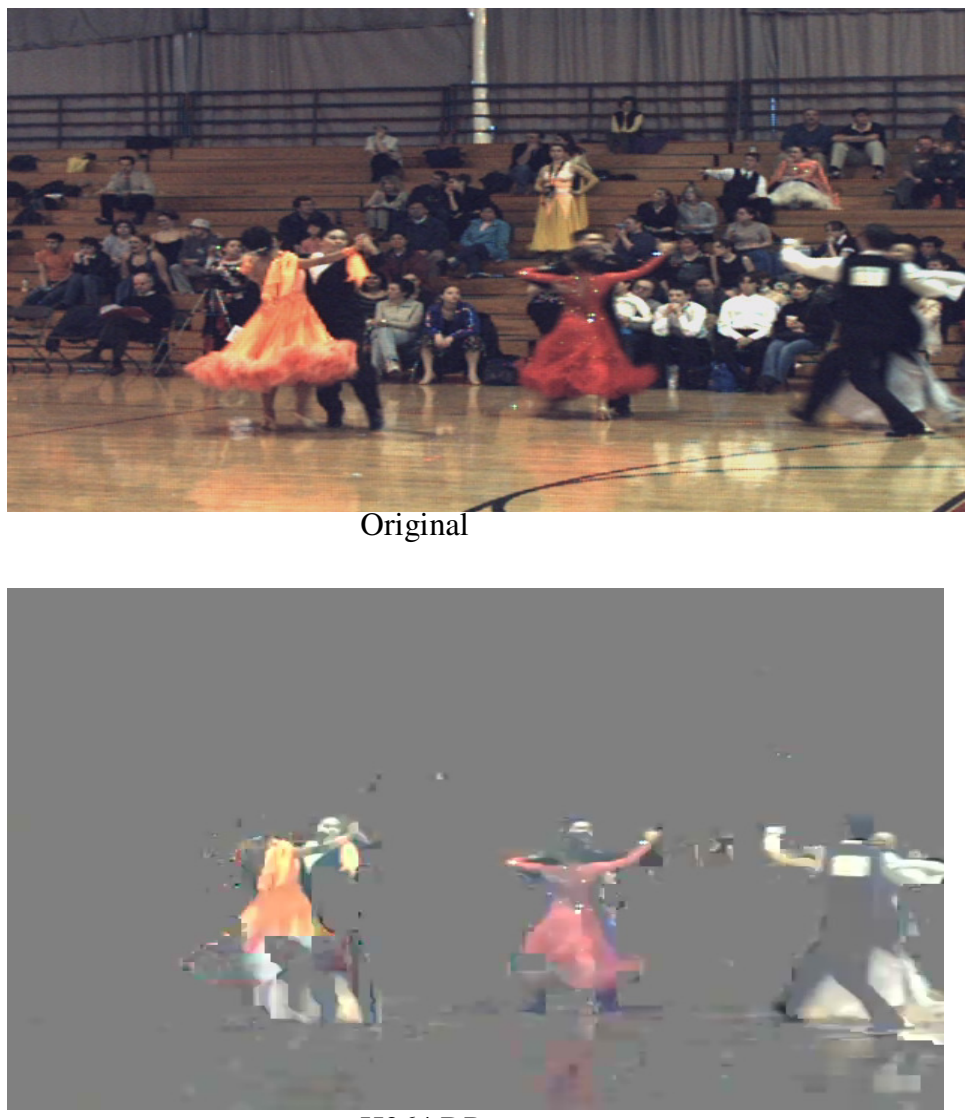

H264 DP

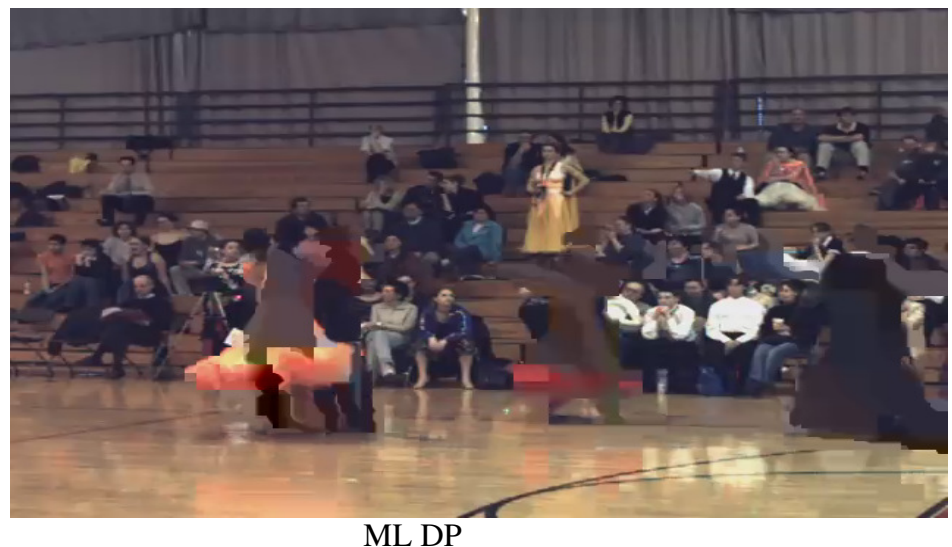

Figure 11. Subjective quality comparison of frame 121 of view 0 at $20 \%$ error rate and $\mathrm{GOP}=16$ for Ballroom sequence. 


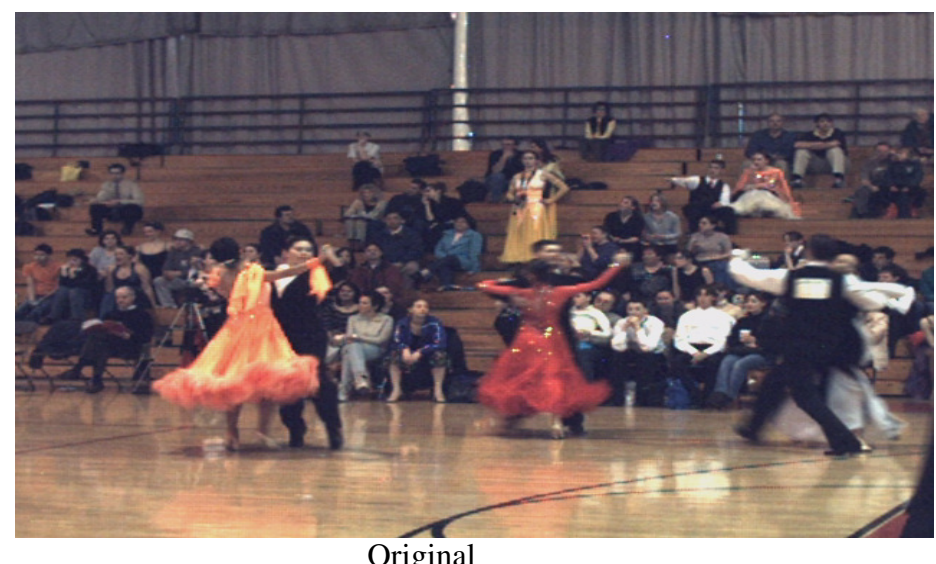

Original

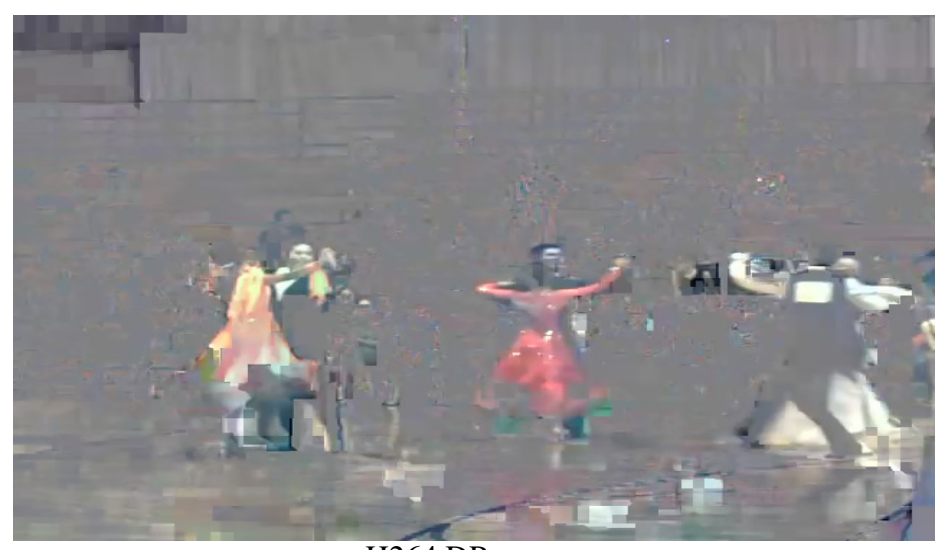

H264 DP

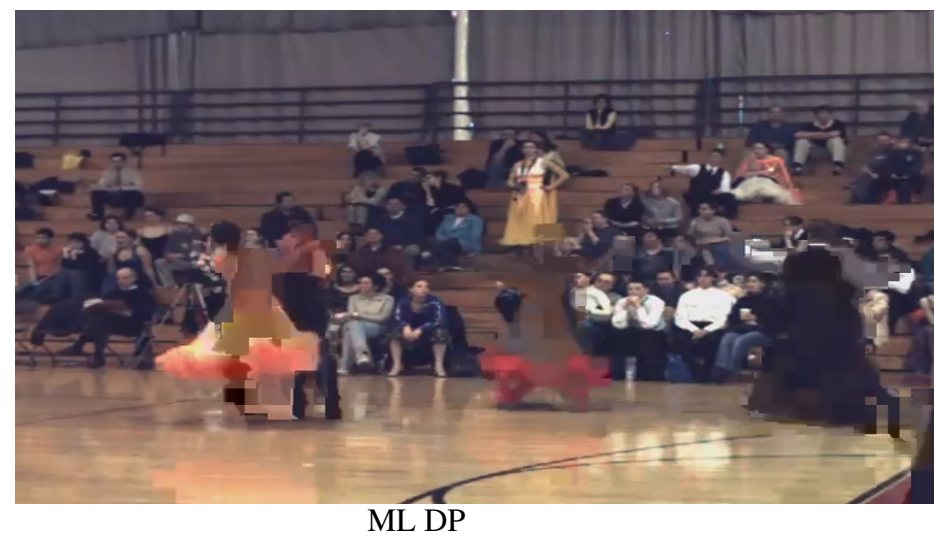

Figure 12 . Subjective quality comparison of frame 121 of view 1 at $20 \%$ error rate and $\mathrm{GOP}=16$ for Ballroom sequence. 

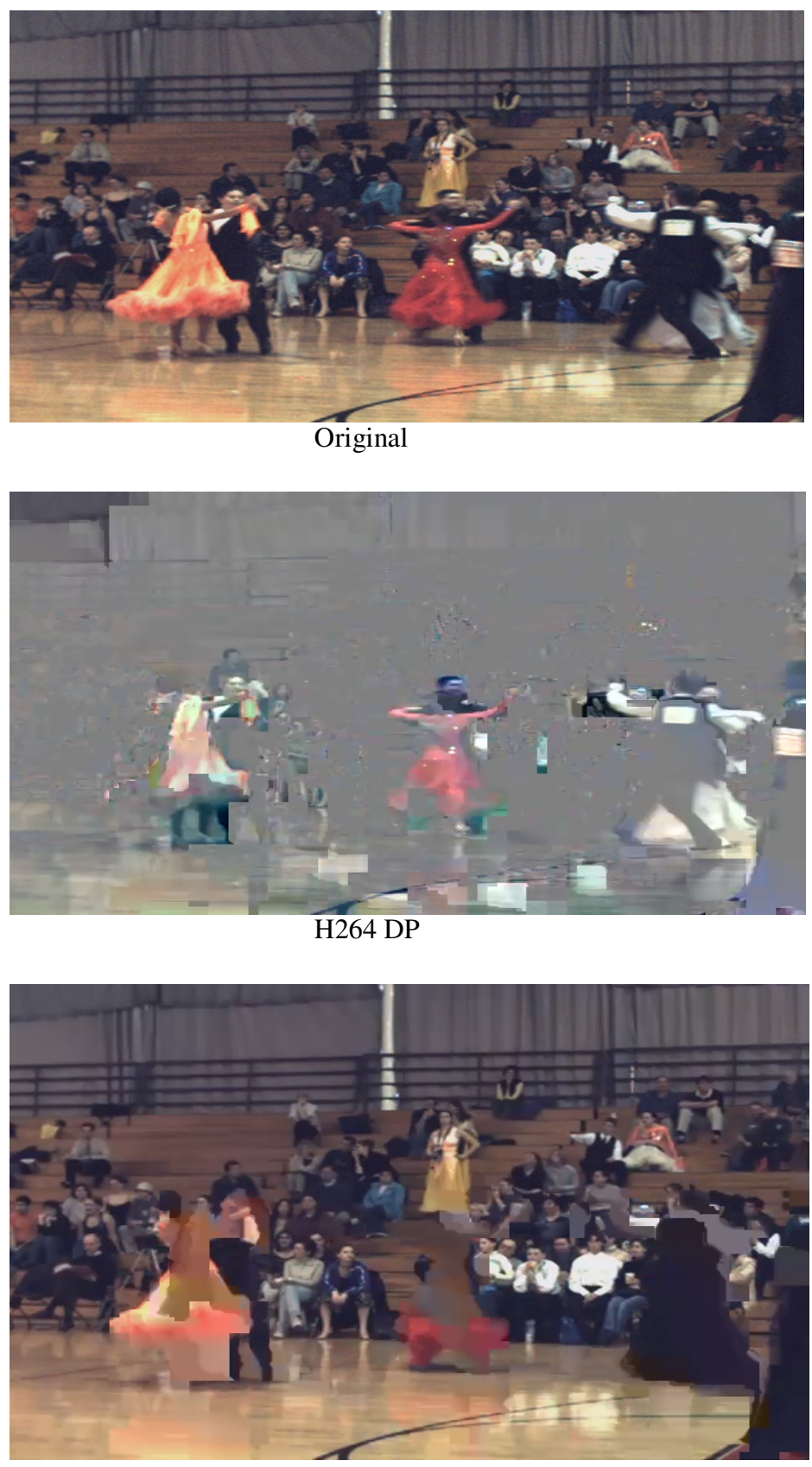

ML DP

Figure 13. Subjective quality comparison of frame 121 of view 2 at $20 \%$ error rate and $\mathrm{GOP}=16$ for Ballroom sequence. 


\section{CONCLusions}

The GOP within a video sequence is one of the key coding parameters that determine the video quality perception of the viewer, more importantly, the GOP size and the motion within the sequence. Large GOP size improves the compression efficiency, which can allow more or higher video content to be transmitted for a given bitrate. However, the effects of error propagation or artefacts due to transmission error in an IP network might be longer. It is necessary to wisely decide what GOP structure and size to support any application such as streaming or transmitting videos. The work in this paper examines the effect of GOP size on erroneous multi-layer data partition bitstream when transmitted over error-prone networks. However, the study in this paper focuses and illustrates the performance of the two algorithms for worst case scenario. Two different techniques namely H264 DP and multi-layer DP are used to demonstrate this effect. Our experimental results illustrate that the Multi-Layer DP technique can improve the visual perception of reconstructed videos for higher error rates within allowable compression efficiency and bitrate. From the results obtained, we can assume and suggest that multi-layer DP technique can suitably be utilized for delivering multiview video content over bandwidth constraint and high error rate channel at a GOP size of 16. Please note that the work in this paper is not claiming to achieve a remarkable visual quality. We are proposing based on simulated results a different approach that can apparently improve the visual quality of multiview video in a very high error rate channel. Part of our future work is to optimize the multi-layer data partitioning technique by implementing error protection technique. The idea is to protect the multiview data from the high error rate in the channel. The decoder error concealment algorithm is going to be extended to employ the hybrid method that can fully exploit the redundancies between macroblocks in both spatial/temporal and interview direction. We anticipate that from our current findings and results, more and better visual quality can be achieved when these techniques are implemented while considering the cost of bit rate and coding efficiency.

\section{ACKNOWLEDGEMENTS}

The authors would like to thank the Petroleum Technology Trust Fund (PTDF) for the research sponsorship.

\section{REFERENCES}

[1] Y. Chen, Y. Wang, K. Ugur, M. M. Hannuksela, J. Lainema and M. Gabbouj, "The emerging MVC standard for 3D video services," EURASIP Journal on Applied Signal Processing, vol. 2009, pp. 8, 2009.

[2] P. A. Akiki and H. W. Maalouf, "A two-stage encoding scheme for holographic data transmission," in Multimedia and Ubiquitous Engineering (MUE), 2011 5th FTRA International Conference on, 2011, pp. 138-142.

[3] M. Ebian, M. El-Sharkawy and S. El-Ramly, "Enhanced dynamic error concealment algorithm for multiview coding based on lost MBs sizes and adaptively selected candidates MBs," in Proceedings of the Fourth International Conference on Signal and Image Processing 2012 (ICSIP 2012), 2013, pp. 435-443.

[4] A. Hermans, "H. 264/MPEG-4 Advanced Video Coding," 2012.

[5] T. Fang and L. Chau, "An error-resilient GOP structure for robust video transmission," Multimedia, IEEE Transactions on, vol. 7, pp. 1131-1138, 2005.

[6] H. Mohib, "End-to-end 3D video communication over heterogeneous networks," 2014.

[7] A. Vetro, J. Xin and H. Sun, "Error resilience video transcoding for wireless communications," Wireless Communications, IEEE, vol. 12, pp. 14-21, 2005.

[8] S. Khan, Y. Peng, E. Steinbach, M. Sgroi and W. Kellerer, "Application-driven cross-layer optimization for video streaming over wireless networks," Communications Magazine, IEEE, vol. 44, pp. 122-130, 2006. 
[9] B. Zatt, M. Porto, J. Scharcanski and S. Bampi, "Gop structure adaptive to the video content for efficient H. 264/AVC encoding," in Image Processing (ICIP), 2010 17th IEEE International Conference on, 2010, pp. 3053-3056.

[10] I. E. Richardson, The H. 264 Advanced Video Compression Standard. John Wiley \& Sons, 2011.

[11] M. Sun, Compressed Video Over Networks. CRC Press, 2000.

[12] L. Al-Jobouri, M. Fleury and M. Ghanbari, "Protecting H. 264/AVC data-partitioned video streams over broadband WiMAX," Advances in Multimedia, vol. 2012, pp. 10, 2012.

[13] S. Wenger, "H. 264/avc over ip," Circuits and Systems for Video Technology, IEEE Transactions on, vol. 13, pp. 645-656, 2003.

[14] A. B. Ibrahim and A. H. Sadka, "Implementation of error resilience technique in multiview video coding," in IEEE Southwest Symposium on Image Analysis and Interpretation, San Diego, California, 2014, pp. 1-4.

[15] Y. Dhondt, S. Mys, K. Vermeirsch and R. Van de Walle, "Constrained inter prediction: Removing dependencies between different data partitions," in Advanced Concepts for Intelligent Vision Systems, 2007, pp. 720-731.

[16] O. Hohlfeld, "Stochastic packet loss model to evaluate QoE impairments," PIK-Praxis Der Informationsverarbeitung Und Kommunikation, vol. 32, pp. 53-56, 2009.

[17] Y. Wang and Q. Zhu, "Error control and concealment for video communication: A review," Proc IEEE, vol. 86, pp. 974-997, 1998.

[18] G. J. Sullivan, P. N. Topiwala and A. Luthra, "The H. 264/AVC advanced video coding standard: Overview and introduction to the fidelity range extensions," in Optical Science and Technology, the SPIE 49th Annual Meeting, 2004, pp. 454-474.

[19] I. Rec, "H. 264 \& ISO/IEC 14496-10 AVC," Advanced Video Coding for Generic Audiovisual Services.ITU-T, 2003.

[20] Z. Lu and H. Yang, Unlocking the Power of OPNET Modeler. Cambridge University Press, 2012.

[21] T. Stockhammer and M. Bystrom, "H. 264/AVC data partitioning for mobile video communication," in Image Processing, 2004. ICIP'04. 2004 International Conference on, 2004, pp. 545-548.

[22] J. Yi, Protocole De Routage À Chemins Multiples Pour Des Réseaux Ad Hoc, 2010.

[23] S. Yasakethu, C. T. Hewage, W. A. C. Fernando and A. M. Kondoz, "Quality analysis for 3D video using 2D video quality models," Consumer Electronics, IEEE Transactions on, vol. 54, pp. 19691976, 2008.

[24] D. Wu, Y. T. Hou and Y. Zhang, "Transporting real-time video over the Internet: Challenges and approaches," Proc IEEE, vol. 88, pp. 1855-1877, 2000.

[25] M. Flierl and B. Girod, "Generalized B pictures and the draft H. 264/AVC video-compression standard," Circuits and Systems for Video Technology, IEEE Transactions on, vol. 13, pp. 587-597, 2003.

[26] A. Aggoun, P. Amon, I. Arbel, A. Chernilov, J. Cosmas, G. Garcia, A. Jari, S. Keller, M. Mattavelli and C. Kontopoulos, "Multimedia delivery in the future internet," 2008.

Abdulkareem Bebeji Ibrahim received the B.ENG. degree in electrical engineering from Bayero University Kano, Nigeria, in 2005, and the MSc. degree in satellite communication and space systems from the University of Sussex, Brighton, United Kingdom, in 2011. He is currently pursuing his $\mathrm{PhD}$. degree in electronic and computer engineering at Brunel University London. His current research interests include error resilience and concealment for 3D multiview video coding and perceptual 3D multiview video quality.

Professor Sadka received the Ph.D. degree in electrical and electronic engineering from Surrey University, Surrey, UK, in 1997. He has nearly 20 years' worth of academic experience and a long track record of scientific leadership in the area of Video Processing and Communications. He is the former Head of the Department of Electronic and Computer Engineering at Brunel University and the Founding Director for the Centre for Media Communications Research.

He has over 200 publications in refereed journals and conferences 3 patents and a

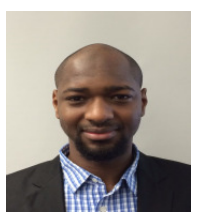
specialised book entitled "Compressed Video Communications" published by Wiley in 2002. To date, he has managed to attract circa $£ 4 \mathrm{M}$ worth of research grants and contracts and has graduated $20 \mathrm{PhD}$ students. He is widely supported by industry and runs his consultancy company VIDCOM. He is a fellow of the IET, a fellow of the HEA and a senior member of the IEEE. 